\title{
Políticas Públicas, MERCOSUR y Educación Superior: la Mercosurización del Estado acreditador ${ }^{1}$
}

Public Policies, MERCOSUR and Higher Education: the Mercosurization of the Accrediting State

Facundo Solanas

CONICET/Instituto de Investigaciones Gino Germani, UBA; GESPH

Universidad Nacional de Mar del Plata fsolanas@yahoo.com
Fecha de recepción:

12.5.18

Fecha de aceptación:

14.8.18

\section{Resumen}

El propósito de este trabajo es dar cuenta de la construcción y continuidad de una política regional en el seno del MERCOSUR y los países asociados, pese a las redefiniciones, los distintos cuestionamientos y crisis por las que atraviesa este proceso de integración. Para ello analizamos la consolidación y expansión de la dinámica de mercosurización del Estado acreditador, retomando trabajos que dan cuenta de la génesis de los primeros instrumentos de políticas públicas regionales, como el Mecanismo Experimental de Acreditación (MEXA) en el MERCOSUR, que en una etapa posterior darían lugar a su traducción en dispositivos permanentes (ARCUSUR), hasta lograr expandirse entre Estados asociados al bloque.

Palabras clave: MERCOSUR - Educación Superior - Acreditación - MEXA - ARCUSUR

\section{Abstract}

The purpose of this paper is to account for the construction and continuity of a regional public policy within 
MERCOSUR and the associated countries, in spite of the redefinitions, the different questions and crises this process of integration is going through. An analysis is conducted of the consolidation and expansion of the mercosurization dynamics of the accrediting State, revisiting previous works that account for the genesis of the first instruments of regional policies, such as the Accreditation Experimental Mechanism (MEXA), at the MERCOSUR level, which eventually evolved into permanent devices (ARCUSUR), to achieve expansion in several member states.

Key-words: MERCOSUR - Higher Education Accreditation - MEXA - ARCUSUR

\section{Introducción}

En los tiempos actuales, pareciera existir cierto consenso para caracterizar la etapa reciente del MERCOSUR como una fase de estancamiento y de redefiniciones. Si las redefiniciones pueden ser consecuencia de los cambios políticos acaecidos en los últimos años en la gran mayoría de los países del bloque, el estancamiento obedecería a trabas que superarían la voluntad política de impulsar una mayor integración. Esta supuesta parálisis responde solo a una lectura unidimensional de la integración: la exclusivamente comercial. Si bien ésta constituye el principal motor en prácticamente todos los procesos de integración, no es el único. A lo largo de su cuarto de siglo de existencia, el bloque ha generado políticas e instrumentos regionales que han logrado superar sus diversas crisis, estancamientos o redefiniciones.

En este trabajo buscamos mostrar la continuidad sectorial que ha mantenido el MERCOSUR en el ámbito educativo desde su creación, independientemente de los cambios políticos y paradigmáticos por los que han atravesado los Estados del bloque, y de las sucesivas redefiniciones, cuestionamientos y supuestas crisis por las que atraviesa o ha atravesado este proceso de integración. Más precisamente, nos proponemos analizar la consolidación y expansión de la dinámica de mercosurización del Estado acreditador (Solanas, 2012). Para ello retomamos trabajos que dan cuenta de la génesis de los primeros instrumentos de políticas públicas regionales, como el Mecanismo Experimental de Acreditación (MEXA) (Solanas, 2015; Botto, 2015; Perrotta, 2014) que en una etapa posterior darían lugar a su traducción en dispositivos permanentes (ARCUSUR), hasta lograr expandirse en distintos Estados asociados al bloque. ${ }^{2}$ 
Para la realización de este trabajo nos apoyaremos en herramientas de análisis provenientes del análisis de políticas públicas. En particular nos focalizaremos sobre cinco dimensiones de análisis: los intereses, las instituciones, las ideas -o 3 i- (Heclo 1994; Hall 2000: Palier y Surel 2005), el territorio y la temporalidad del proceso -o $2 t-$, así como en fuentes primarias y secundarias, específicamente en entrevistas semiestructuradas con informantes claves y fuentes documentales.

El trabajo se estructura en cinco partes. En la primera, presentaremos nuestras herramientas de análisis, definiremos la noción de mercosurización a partir de la expansión que ha tenido el Estado acreditador en Suramérica y daremos cuenta de las tres temporalidades por las que ha atravesado este proceso. En la segunda analizaremos el primer tiempo de la dinámica de mercosurización. En la tercera, abordaremos la segunda temporalidad. En la cuarta parte, consecuentemente analizaremos el tercer tiempo de este proceso. En la última abordaremos los impactos que ha tenido esta dinámica, para cerrar con unas reflexiones.

\section{Definición de nuestras herramientas de análisis}

Retomando algunos de los trabajos evocados precedentemente, el presente focaliza tres variables de análisis principales: los intereses, las instituciones, las ideas -o tres i- (Heclo 1994; Hall 2000: Palier y Surel 2005) que serán “cruzadas” con otras dos dimensiones: el tiempo y el territorio -o dos t. Movilizar conjuntamente estas dimensiones implica que estas variables explicativas no son excluyentes unas de otras, pero pueden asociarse para delimitar los ejes de la investigación y las dinámicas pertinentes en el análisis del Estado en acción (Palier y Surel, 2005: 10). La posibilidad de articular estas variables nos permite delimitar nuestro objeto y apoyarnos de diferentes maneras sobre su poder explicativo según las características propias de cada proceso y los diversos momentos de su evolución. Por lo general, cada una de estas dimensiones de análisis siguen una secuencia propia: las ideas se desarrollan a partir de tiempos largos, dado que son las menos susceptibles de cambiar en el corto plazo; los intereses se caracterizan por los tiempos cortos, dado que frecuentemente se definen en la interacción; en tanto que las instituciones cuentan con una temporalidad intermedia, producto de la efectiva lógica de institucionalización de los dispositivos de acción pública (id. 29-30).

A partir de la importancia creciente de los organismos multilaterales sobre la política nacional de los Estados miembro del bloque, así como sobre diferentes políticas regionales, hemos decido jerarquizar a modo de sub-variable transversal a las 3i, la dimensión internacional o cuarta sub-i.

Las dimensiones de análisis que tomamos en cuenta se circunscriben a determinado tiempo y espacio, en donde el espacio se traduce en territorio, producto de los procesos de la incorporación nuevos Estados al bloque -principalmente con carácter de Estados asociados. Por lo tanto, la dimensión territorial aparecerá como una variable de análisis fundamental del proceso de mercosurización, que al igual que las $3 i$ será analizada a lo largo de tres temporalidades. 
El primer tiempo comienza con la génesis del Sector Educativo del MERCOSUR (SEM) con la creación de las Reuniones de Ministros de Educación (RME) en 1991 (el mismo año de su constitución como bloque) e incluye la firma de los primeros protocolos y debates con vistas a facilitar el reconocimiento de los títulos universitarios con fines académicos y profesionales dentro del bloque. Y culmina con la definición del memorándum de entendimiento en 1998, cuando se decide la creación del primer instrumento de acreditación regional, el MEXA, que se circunscribe a los aspectos exclusivamente académicos del reconocimiento.

El segundo tiempo comienza en 1998 con el nacimiento del MEXA y su implementación. Este también implicó una redefinición de objetivos e instrumentos de reconocimiento a partir de la movilización de algunos actores no gubernamentales y se prolonga desde su aplicación hasta su expansión con el compromiso de implementación del ARCUSUR en 2006.

El tercer tiempo se inicia en el período comprendido entre el año 2006 con la aplicación del ARCUSUR y se expande hasta el presente (Cuadro $N^{\circ} 1$ ).

Cuadro $\mathrm{N}^{\circ}$ 1: Las temporalidades de la Mercosurización del Estado acreditador

\begin{tabular}{|c|c|}
\hline Períodos & Acreditación regional \\
\hline 1991-98: Definición & Creación del SEM, primeros protocolos sectoriales y decisión de la acreditación \\
\hline 1998-2006: Transición & $\begin{array}{c}\text { Implementación del MEXA y decisión de avanzar hacia un dispositivo } \\
\text { permanente }\end{array}$ \\
\hline 2006-actual & Implementación, consolidación y expansión de ARCUSUR \\
\hline
\end{tabular}

La noción de mercosurización concierne al conjunto de procesos de ajustes institucionales, estratégicos, normativos y cognitivos, primero introducidos en la lógica del discurso (nacional y subnacional), las estructuras políticas internas y las políticas públicas de los principales Estados miembros del MERCOSUR o de sus países asociados, a través de la influencia de organizaciones internacionales y, luego, incorporadas en el proceso de construcción de la política regional (Solanas 2015).

Pasaremos a analizar esta dinámica de mercosurización en cada una de sus dimensiones secuencialmente a lo largo de sus tres temporalidades.

\section{Primer tiempo: De la génesis a la definición}

El tratado de Asunción, de naturaleza exclusivamente comercial, preveía la libre circulación de los "factores productivos". Bajo este amplio concepto, donde aparecen comprendidos los profesionales, los docentes e investigadores, invitaba indirectamente a introducir en la agenda institucional la cuestión educativa y la validez de las formaciones. 
De este modo, las autoridades de los ministerios de Educación del bloque se reúnen al poco tiempo de suscripto el tratado y firman el Protocolo de Intenciones en diciembre de 1991. De este modo, se creaban las Reuniones de Ministros de Educación del MERCOSUR que se institucionalizarían en un conjunto de comisiones y grupos de trabajo (véase gráfico $\mathrm{N}^{\circ} 1$ ).

Gráfico N¹ GT: Grupo de Trabajo

\section{ESTRUCTURA DEL MERCOSUR}

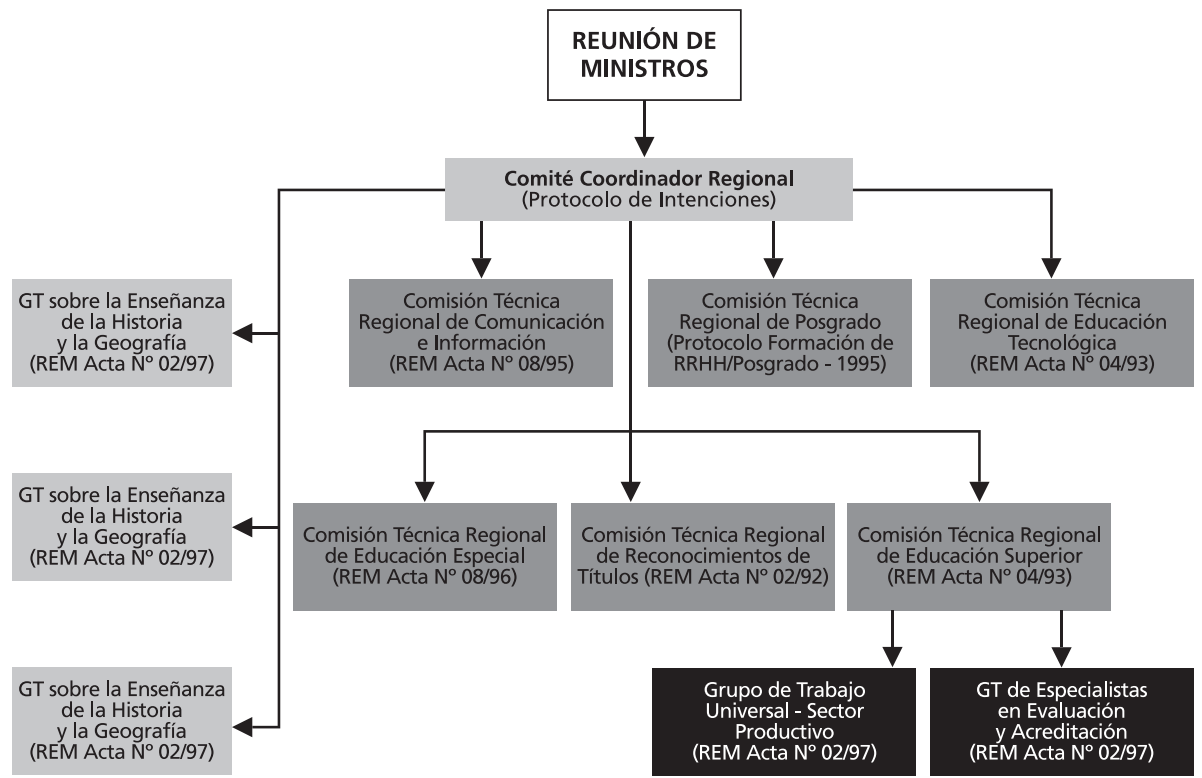

Fuente: SEM

Si los actores motores de este proceso eran fundamentalmente los ministerios de Educación, también diversas asociaciones y colegios profesionales habían manifestado su interés en avanzar hacia la integración del espacio profesional del bloque.

Teniendo en cuenta la naturaleza fuertemente intergubernamental del proceso de integración, la agenda regional sería fuertemente permeable a los cambios que tendrían lugar en los Estados del bloque. Para ese entonces comenzaba a instalarse en la agenda argentina de educación superior la necesidad de una reforma que comprendiese determinados dispositivos de evaluación y acreditación de las carreras universitarias de grado y postgrado. Como ya ha sido abordado en otros trabajos, ello había constituido una transferencia por parte de organismos internacionales como el Banco Mundial (BM) que en sus informes venia recomendando la adopción de estos instrumentos 
(Chiroleau, 2000; Solanas, 2012). La creación de la Comisión Nacional de Evaluación y Acreditación Universitaria (CONEAU), marcaría un giro en las discusiones que se venían dando dentro del SEM. Si en principio la reforma y la creación de esta institución había sido fuertemente resistida, al poco tiempo pasaría a ser aceptada por la mayor parte de los actores universitarios, dado que pondría freno al proceso de apertura indiscriminado de universidades de dudosa calidad.

La idea de resolver la calidad de las formaciones antes que la validez de los diplomas cobra fuerza, y la incorporación de nuevos Estados asociados al bloque, como Chile y Bolivia, sería decisiva para apuntalar la postura argentina inclinada hacia este camino. Por lo tanto, luego de evaluar distintas propuestas se decide que la acreditación regional de carreras constituya la vía para el reconocimiento de las titulaciones.

En 1998, se firmaría el Memorándum de Entendimiento entre los Estados miembros (4) y los asociados (2), que marcaría el inicio de la implementación de este instrumento.

\section{Segundo tiempo: La transición}

La decisión de avanzar hacia los dispositivos de acreditación regional de carreras de grado, impulsaría la dinámica de mercosurización y expansión del Estado acreditador con la creación de nuevas agencias en otros países. Chile sería el primer país en crear su agencia, pero le seguiría Paraguay, mientras que los demás Estados optarían en principio por suplir esa carencia con dispositivos alternativos, como Brasil y Uruguay. Por su parte Bolivia haría acreditar sus carreras por la CONEAU argentina (Solanas, 2012, Véase Cuadro $N^{\circ} 2$ ), cuyos estándares de acreditación serían tenidos en cuenta como referencia principal.

\section{Cuadro $\mathrm{N}^{\circ} 2$ : Implementación del MEXA en 6 países}

\begin{tabular}{|c|c|}
\hline País & Institución de acreditación \\
\hline Argentina & CONEAU (1996) \\
\hline Brasil & Secretaría de Educación Superior (SESU) \\
\hline Paraguay & ANEAES (2003) \\
\hline Uruguay & Comisiones ad hoc \\
\hline Chile & Comisión Nacional de Acreditación de Pregrado (CNAP, 1999) \\
\hline Bolivia & Delega en la CONEAU \\
\hline
\end{tabular}

Como su nombre lo indica, el carácter "experimental" del MEXA compatibilizaba con la cautela de ciertos países acerca de los posibles pasos a dar antes de crear una agencia de acreditación propia. A pesar de ello, los cuatro países miembros del MERCOSUR y sus dos asociados acreditaron, en su mayor parte, las tres carreras que comprendía el MEXA. 
La idea de acreditación comienza a cobrar mayor legitimidad en el seno de los Estados miembro, aún en países donde había sido fuertemente resistida por los actores universitarios, como Argentina. A su vez, la necesidad de crear una institución de estas características comienzó a ser aceptada por la mayor parte de los Estados del bloque.

En el caso argentino, si la creación de una institución de acreditación había sido fruto de una transferencia transnacional-nacional (Evans y Davies 1999), a partir de los informes provenientes del BM cuyos diagnósticos planteaban la necesidad de evaluar el sistema universitario y acreditar sus carreras (Solanas 2012), esta institución se vuelve un aliado de las principales instituciones de educación superior que la habían cuestionado. Producto de la mercantilización y germinación de universidades de diverso tipo, la existencia de la CONEAU -a diferencia de buena parte de sus principales países vecinos- contribuyó a frenar ese proceso de apertura indiscriminado de universidades.

Las universidades han participado de diversas maneras de este proceso. En primer lugar, ofreciendo voluntariamente sus carreras a fin de que se sometan al proceso de acreditación. En segundo lugar, aportando el conocimiento de sus docentes e investigadores quienes en distintos casos participarían del establecimiento de los estándares de calidad que requiere este proceso, o bien se convertirían en pares evaluadores de las carreras presentadas para ser acreditadas.

La estructura organizacional del SEM sufrirá algunas modificaciones en consecuencia con las comisiones y grupos de trabajo que mantienen una continuidad y progresan en la implementación de determinadas políticas o instrumentos de políticas.

Una vez concluida la implementación del MEXA, los países participantes decidieron continuar el proceso transformando el instrumento experimental en un dispositivo permanente, creando el ARCUSUR.

\section{Tercer tiempo: De la continuidad a la expansión}

La aprobación del ARCUSUR tendrá un gran impacto sobre la expansión de la dinámica de mercosurización del Estado acreditador. El ARCUSUR será ratificado por el Consejo del Mercado Común del Mercosur (CMC) dándole un mayor reconocimiento institucional. Los países participantes que hasta ese momento no contaban con agencias de acreditación, como Brasil, crean su propia institución y aquellos que aún no lo hicieron, como Uruguay y Bolivia cuentan con proyectos de ley que aguardan la aprobación parlamentaria para proceder a la creación de su agencia.

Además, en el caso de Bolivia, la Comisión Nacional de Acreditación de Carreras Universitarias - CNACU, legalmente constituida por Ley 070 de 20 de diciembre 
de 2010, tiene la función de Evaluar y Acreditar la Educación Superior Universitaria Pública y Privada, apuntando a coordinar procesos de Evaluación para la Acreditación y Certificación de la calidad educativa en el marco del Sistema ARCUSUR.

La dinámica de mercosurización no se limita a la creación de instituciones de acreditación en los países participantes del proceso, en sintonía con las transferencias cognitivas que difunden e incorporan la necesidad de acreditar las distintas carreras entre las autoridades educativas de los países de la región. Como consecuencia del impulso que han tenido estas políticas en el bloque, otros países asociados al MERCOSUR se incorporan a esta dinámica. Como el caso de Colombia, que contaba con la primer agencia de acreditación de la región, Venezuela que transforma su propia institución y la última incorporación ha sido la de Ecuador cuya institución ha sido creada recientemente (Véase Cuadro $\mathrm{N}^{\circ}$ 3). La dimensión territorial cobra un peso cualitativo importante, dado que estos Estados comienzan a participar e interesarse por el proceso, pese a que por diferentes razones aún no han logrado acreditar carreras de grado en el seno del ARCUSUR.

\section{Cuadro No3: El ARCUSUR y las transformaciones institucionales}

\begin{tabular}{|l|l|}
\hline País & Institución de acreditación \\
\hline Argentina & CONEAU (1996) \\
\hline Brasil & $\begin{array}{l}\text { Sistema Nacional de Avaliação da Educação Superior (SINAES) } \\
y \text { Comissão Nacional de Avaliação da Educação Superior (CONAES), (2004) }\end{array}$ \\
\hline Paraguay & ANEAES (2003) \\
\hline Uruguay & $\begin{array}{l}\text { Estan consensuando proyecto de ley que crea la “Agencia para la Promoción y Aseguramiento } \\
\text { de la Calidad de la Educación Terciaria" (APACET) }\end{array}$ \\
\hline Chile & Comisión Nacional de Acreditación (CNA) que supliría a la CNAP (2006) \\
\hline Bolivia & $\begin{array}{l}\text { Creó la Comisión Nacional de Acreditación de Carreras Universitarias CNACU y se encuentra } \\
\text { discutiendo la creación de una Agencia Plurinacional de Evaluación y Acreditación de la Educación } \\
\text { Superior Universitaria (APEAESU) }\end{array}$ \\
\hline Colombia & $\begin{array}{l}\text { Cuenta con una institución acreditadora: el Consejo Nacional de Acreditación dependiente } \\
\text { del Consejo Nacional de Educación Superior (CESU) (1992) }\end{array}$ \\
\hline Venezuela & $\begin{array}{l}\text { Consejo Consultivo de Postgrado (1983-1996); Comité de Evaluación y Acreditación de Programas } \\
\text { e Instituciones de Educación (CEAPIES) (2008) }\end{array}$ \\
\hline Ecuador & $\begin{array}{l}\text { Consejo de Evaluación, Acreditación y Aseguramiento de la Calidad de la Educación } \\
\text { Superior - CEAACES (2012) }\end{array}$ \\
\hline
\end{tabular}

Debido al impulso que ha tenido esta dinámica por un lado, al estancamiento que han tenido otras en el seno del SEM, por otro lado, así como a la necesidad de redefinir sectores, se producen nuevas transformaciones en el sector que se visualizaran en la nueva arquitectura institucional del sector (véase gráfico $\mathrm{N}^{\circ} 2$ ). 


\section{Gráfico $N^{\circ}$ 2: Nueva Estructura Institucional del SEM}

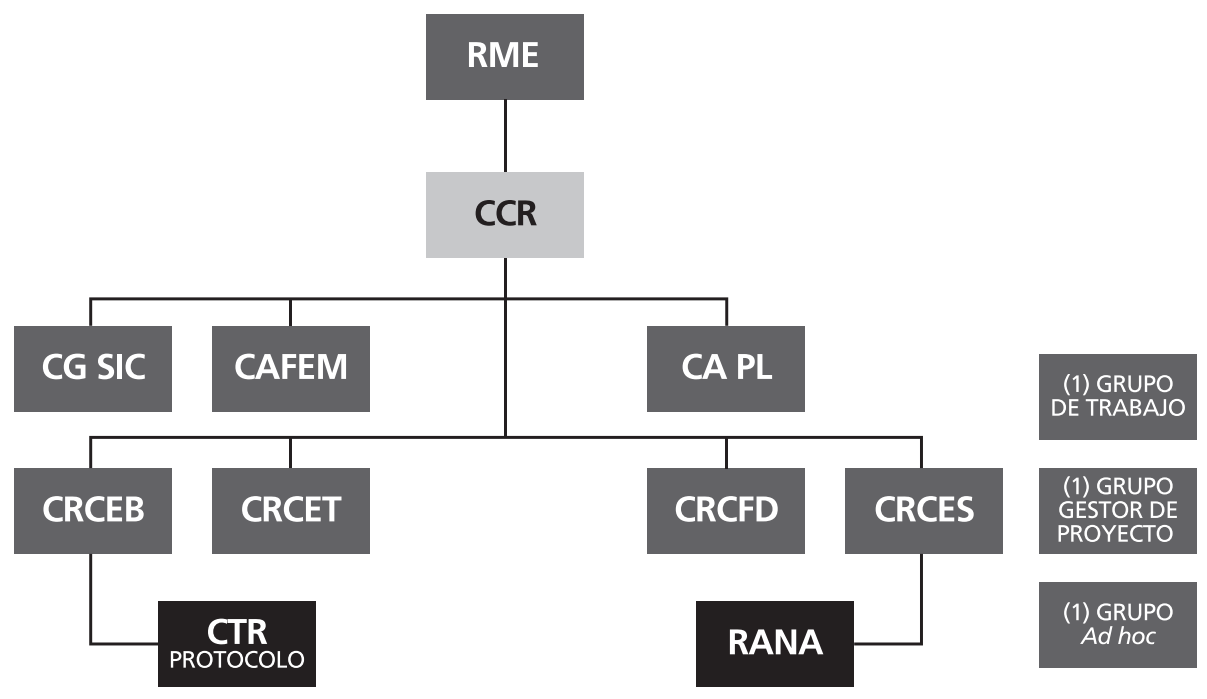

Para que esta dinámica de mercosurización haya sido posible, es necesario destacar la participación activa de los actores universitarios, quienes deciden participar voluntariamente del ARCUSUR, seleccionando las carreras que se someterán a este dispositivo regional. Son pares evaluadores pertenecientes a universidades quienes son convocados a fin de establecer los estándares de calidad y luego proceder a la evaluación de cada carrera.

\section{Impacto de la mercosurización por tiempos}

Tomando en cuenta los tres tiempos analizados y a modo recapitulativo, podemos dar cuenta de la forma en que la dinámica de mercosurización se ha venido consolidando. Si en el primer tiempo, de definiciones, existía una sola agencia de acreditación y la participación de países aumentaba de 4 a 6 , el lanzamiento del primer instrumento regional: el MEXA contribuiría a crear instituciones en la mayor parte de los países, pasando de 1 a 4 agencias de acreditación. Finalmente, el tercer tiempo caracterizado por el pasaje a un dispositivo permanente -y no experimental-, fomentaría la expansión territorial del instrumento, con la incorporación de otros tres miembros y consolidaría la dinámica de mercosurización del Estado acreditador con la creación de otras instituciones y los proyectos de creación en la totalidad de países participantes del ARCUSUR (Véase Cuadro N4). 


\section{Cuadro $\mathrm{N}^{\circ} 4$ : Impacto de la mercosurización por tiempos}

\begin{tabular}{|c|c|c|}
\hline Periodos & Países participantes & Cantidad de instituciones de acreditación \\
\hline Definición & De 4 a 6 & 1 \\
\hline Transición & 6 & 4 \\
\hline Consolidación & De 6 a 9 & $7+2$ proyectos \\
\hline
\end{tabular}

Los actores universitarios que durante el primer tiempo se habían opuesto al Estado acreditador por diferentes motivos -entre ellos por la participación de determinados organismos transnacionales como el Banco Mundial-, comienzan a percibir las ventajas de la existencia de una institución de estas características y participan del proceso.

La idea del Estado acreditador paulatinamente va siendo incorporada en el conjunto de países miembros o asociados al MERCOSUR que comienzan a crear sus instituciones, en buena parte de los casos con vistas a participar del MEXA y del ARCUSUR. La continuidad de este instrumento permitirá expandir territorialmente el impacto de la mercosurización entre los últimos países incorporados como Estados asociados al bloque.

\section{Reflexiones finales}

Independientemente de la coyuntura y la tensión política existente en este momento, el proceso de mercosurización del Estado acreditador surgido en los años noventas fue evolucionando y consolidándose en forma ininterrumpida, todo lo que haría pensar que el proceso proseguirá su implementación e inclusive continúe expandiéndose.

La dinámica de mercosurización del Estado acreditador se muestra como un elemento de continuidad, pese a los vaivenes políticos que experimentan los Estados miembros del bloque. Sin dudas ello es consecuencia de la despolitización de un instrumento que permaneció ajeno a la politización imperante en la mayoría de los Estados de la región, pero que como buena parte de las políticas del MERCOSUR carece de visibilidad frente a la agenda mediática.

Políticas regionales e instrumentos de políticas como las desarrolladas por el SEM trascienden el estancamiento o la parálisis económica que experimenta el intercambio comercial en diferentes sectores intra-bloque y, si bien solas no bastan para impulsar las interdependencias necesarias que requiere este proceso de integración para profundizarse, demuestran que los efectos e impactos que ha provocado el MERCOSUR no se ciernen exclusivamente a la agenda comercial. Sus efectos son capaces de asegurar continuidades por sobre los ritmos comerciales y su dinámica de mercosurización se posiciona más allá de los diferentes cambios políticos experimentados por los países del bloque. 
${ }^{1}$ Este artículo se basa en una ponencia presentada en las Terceras Jornadas Internacionales: Sociedad, Estado y Universidad: "La universidad en el siglo XXI: La educación como derecho y las dimensiones de la inclusión social", realizadas el 30 de Noviembre, 1 y 2 de Diciembre de 2017, Mar del Plata.

${ }^{2}$ Por instrumento de políticas públicas entendemos a los dispositivos técnicos y sociales que organizan las relaciones sociales específicas entre el poder público y sus destinatarios en función de las representaciones y significaciones que éste entraña (Lascoumes y Le Galès 2004). 


\section{Referencias bibliográficas}

Camou,Antonio (2007) “Los «juegos» de la evaluación universitaria en la Argentina. Notas sobre las interacciones conflictivas entre Estado y universidad”. Evaluando la evaluación: políticas universitarias, instituciones y actores en Argentina y América Latina. Pedro Krotsch, Antonio Camou, Marcelo Prati (Coords.). Buenos Aires: Prometeo Libros, pp. 29-68.

Chiroleu, Adriana (2000) "La Educación Superior en los países del Mercosur. El reconocimiento de las singularidades como punto de partida de la integración regional". Revista de la Educación Superior,Vol. XXIX (115) 1-10.

Botto, Mercedes (2015). "La transnacionalización de la educación superior: ¿qué papel juegan los nuevos regionalismos en la difusión de estas ideas? El caso del Mercosur (1992-2012) en perspectica comparada". Revista Iberoamericana de Educación Superior (RIES), vol.VI, No16:90-109.

Evans, Mark y Jonathan Davies (1999) “Interpretación de la transferencia de políticas: una perspectiva multidisciplinaria y de niveles múltiples”. Gestión y Política Pública Vol.VIII (2), segundo semestre, 69-199.

Lascoumes, Patrick y Pierre Le Galès, dirs. (2004). Gouverner par les instruments. Paris: Presses de la Fondation Nationale des Sciences Politiques.

Hall, Peter (2000) "Le Rôle des intérêts des institutions et des idées dans l'économie politique comparée des pays industrialisés". Revue Internationale de politique comparée, 7 (1). 53-92.

Palier, Bruno, Yves Surel et al. (2007) L'Europe en action. L'européanisation dans une perspective comparée. Paris: L'Harmattan.

Palier, Bruno e Yves Surel (2005) "Les "Trois i " et l'analyse de l'état en action". Revue française de science politique. vol. 55, (1): 7-32.

Perrotta, Daniela (2014) "La educación superior en el MERCOSUR: la acomodación entre las políticas domésticas y la política regional”. Revista Perspectivas de Políticas Públicas, Año 3 N6: 177-206. 
Solanas, Facundo (2012) "El Estado Acreditador: Del Caso Argentino al MERCOSUR”. Denise Leite, Maria Elly Herz Genro, Facundo Solanas, Vivian Fiori, Raúl Alberto Álvarez Ortega. Políticas de evaluación universitaria en América Latina: perspectivas críticas. Buenos Aires: CLACSO/IIGG:99-138. también disponible en: http://bibliotecavirtual.clacso.org.ar/clacso/posgrados/20120914120239/ PoliticasdeEvaluacionUniversitaria.pdf

Solanas, Facundo (2015) "De la europeización a la mercosurización de políticas públicas: Una comparación del proceso de Bolonia europeo y el Estado acreditador en el MERCOSUR", Política Pública, Revista del Departamento Gestión y Política Pública, Vol. 8 (2), Universidad de Santiago de Chile, pp 43-72.

\section{Cómo citar este artículo}

Solanas, Facundo (2019) "Políticas Públicas, MERCOSUR y Educación Superior: la Mercosurización del Estado acreditador”. Revista Perspectivas de Políticas Públicas vol. $8 \mathrm{~N}^{\circ} 16: 427-439$ 